\title{
Tunable on chip optofluidic laser
}

Bakal, Avraham; Vannahme, Christoph; Kristensen, Anders; Levy, Uriel

Published in:

Applied Physics Letters

Publication date:

2015

Document Version

Publisher's PDF, also known as Version of record

Link back to DTU Orbit

Citation (APA):

Bakal, A., Vannahme, C., Kristensen, A., \& Levy, U. (2015). Tunable on chip optofluidic laser. Applied Physics Letters, 107(21), [211105].

\section{General rights}

Copyright and moral rights for the publications made accessible in the public portal are retained by the authors and/or other copyright owners and it is a condition of accessing publications that users recognise and abide by the legal requirements associated with these rights.

- Users may download and print one copy of any publication from the public portal for the purpose of private study or research.

- You may not further distribute the material or use it for any profit-making activity or commercial gain

- You may freely distribute the URL identifying the publication in the public portal

If you believe that this document breaches copyright please contact us providing details, and we will remove access to the work immediately and investigate your claim 


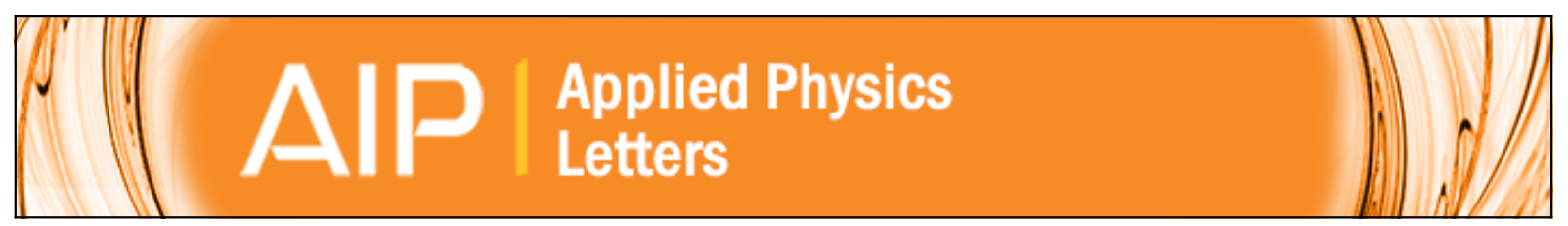

\section{Tunable on chip optofluidic laser}

Avraham Bakal, Christoph Vannahme, Anders Kristensen, and Uriel Levy

Citation: Applied Physics Letters 107, 211105 (2015); doi: 10.1063/1.4936235

View online: http://dx.doi.org/10.1063/1.4936235

View Table of Contents: http://scitation.aip.org/content/aip/journal/apl/107/21?ver=pdfcov

Published by the AIP Publishing

\section{Articles you may be interested in}

Tunable single mode lasing from an on-chip optofluidic ring resonator laser

Appl. Phys. Lett. 98, 061103 (2011); 10.1063/1.3554362

Pneumatically tunable optofluidic dye laser

Appl. Phys. Lett. 96, 081101 (2010); 10.1063/1.3324885

Optofluidic ring resonator based dye laser

Appl. Phys. Lett. 90, 221101 (2007); 10.1063/1.2743884

Optofluidic third order distributed feedback dye laser

Appl. Phys. Lett. 89, 103518 (2006); 10.1063/1.2345602

Microfluidic tunable dye laser with integrated mixer and ring resonator

Appl. Phys. Lett. 86, 264101 (2005); 10.1063/1.1968421

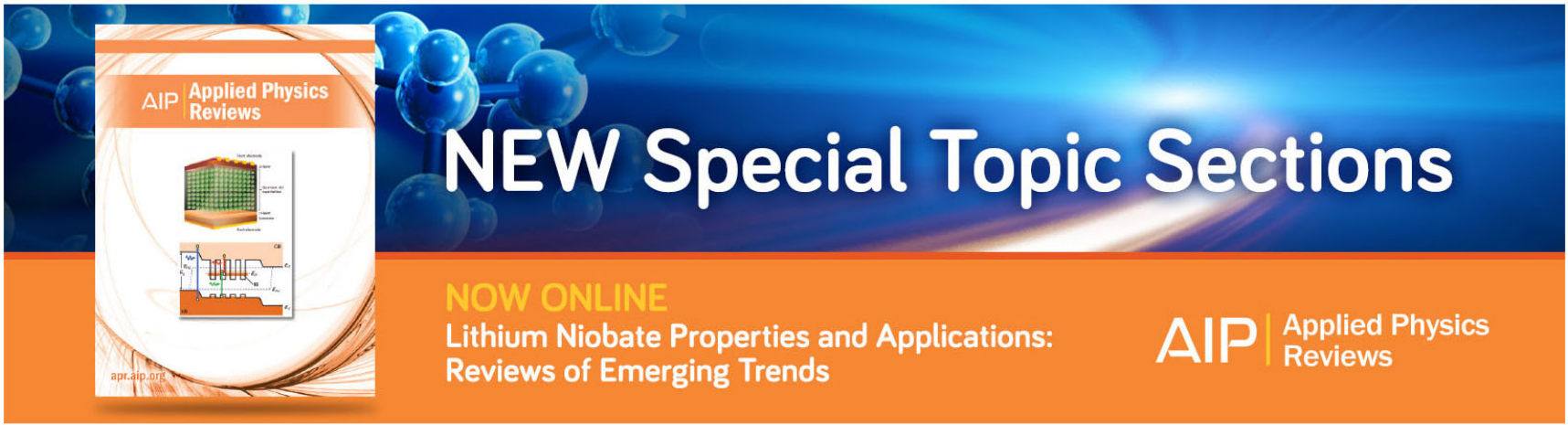




\title{
Tunable on chip optofluidic laser
}

\author{
Avraham Bakal, ${ }^{1, a)}$ Christoph Vannahme, ${ }^{2}$ Anders Kristensen, ${ }^{2}$ and Uriel Levy ${ }^{1, b)}$ \\ ${ }^{1}$ Department of Applied Physics, The Benin School of Engineering, The Center for Nanoscience \\ and Nanotechnology, The Hebrew University of Jerusalem, Jerusalem 91904, Israel \\ ${ }^{2}$ Department of Micro- and Nanotechnology, Technical University of Denmark, DTU Nanotech, \\ $\emptyset$ rsteds Plads, Building 345E, DK-2800 Kgs. Lyngby, Denmark
}

(Received 4 September 2015; accepted 10 November 2015; published online 23 November 2015)

A chip scale tunable laser in the visible spectral band is realized by generating a periodic droplet array inside a microfluidic channel. Combined with a gain medium within the droplets, the periodic structure provides the optical feedback of the laser. By controlling the pressure applied to two separate inlets we can change the period of the droplet array. As a result, the lasing frequency is tuned over a broad spectral range. Using this configuration, we demonstrate wavelength tunability of about $70 \mathrm{~nm}$ and lasing threshold of about $15 \mu \mathrm{J} / \mathrm{mm}^{2}$. ( 2015 AIP Publishing LLC.

[http://dx.doi.org/10.1063/1.4936235]

The synergy of microfluidics and photonics has sparked the term "Optofluidics." Taking advantage of flexibility and high degree of integration, alongside easy of fabrication, optofluidic devices, and systems are becoming vital in a large variety of applications spanning from sensing, cell sorting, and sequencing to tunable optical devices. ${ }^{1-3}$ Among the various optofluidic components, the optofluidic dye laser holds great promise as a critical component in future integrated photonic systems, primarily pertaining lab on chip applications. ${ }^{4-6}$ Over the last 15 years, a tremendous progress in the field of optofluidic lasers has been reported, driven by the rapid advancements in microfabrication and microfluidic technologies, alongside the growing need for lab on chip applications.

Several different configurations of optofluidic dye lasers have been demonstrated based on, e.g., Fabry-Perot cavities, ${ }^{7}$ embedded Distributed Feedback (DFB) gratings, ${ }^{8-13}$ whispering gallery modes in droplets, ${ }^{14-17}$ and ring resonators. ${ }^{18,19}$ In these lasers, the gain medium is in a fluidic form. A complementary approach is the immersion of the dye within polymers in solid form. ${ }^{6,20}$ Typically, the optofluidic lasers consist of a dye mixed within a solvent as a gain medium. An important advantage of the optofluidic technology resides in its capability of realizing adaptive optical devices. ${ }^{3}$ For example, it is possible to replace the dye or the solvent in the optofluidic laser for the purpose of controlling its lasing characteristics, ${ }^{11}$ and achieve the desired property of wavelength tunability. Indeed, over the last few years several on chip tunable optofluidic lasers were demonstrated, e.g., by combining two different dye molecules in a solvent ${ }^{21,22}$ or by taking advantage of the high elasticity of poly-dimethyl-siloxane (PDMS) in order to tune the periodicity of a Bragg grating. ${ }^{23}$

In this paper we describe a robust and flexible tuning mechanism which enables us to achieve a chip scale optofluidic tunable laser operating on the basis of embedding a periodic droplet array within a microfluidic channel (Fig. 1). Our goal is to construct a tunable DFB structure supporting lasing

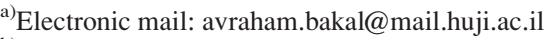

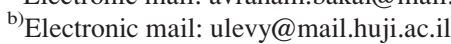

at a given wavelength which can be selected at will over a broad spectral range. For this purpose, a micro-fluidic channel is constructed with a flow of periodically arranged droplets (Benzyl alcohol + Rhodamine 6G ${ }^{24,25}$ (R6G)) immersed in mineral oil. The periodic structure is acting as an optical feedback.

Additional mechanisms such as whispering gallery modes are excluded by choosing the droplet size to be sufficiently small. A micrograph of our constructed optofluidic device together with a schematic drawing showing the device configuration that enables the generation of periodic droplet array within a microfluidic channel is depicted in Fig. 2. The device is based on a concept described by Beatus et al. ${ }^{26}$ where the dimensions and operation parameters have been optimized for our application. We have previously used this concept for measuring the flow rate of microfluidic channels taking advantage of the Doppler effect. ${ }^{27}$ The device is fabricated using standard soft-lithography technique. It is made of PDMS and consists of two inlets and one outlet. One of the inlets is designed for delivering the gain medium, namely, R6G dissolved in benzyl alcohol (refractive index of $\mathrm{n} \sim 1.56$ ) while the other delivers the oil which has a lower refractive index $(\mathrm{n} \sim 1.46)$. The two channels operate under conditions of continuous flow and are connected via a $\mathrm{T}$ junction into a unified channel, where benzyl alcohol

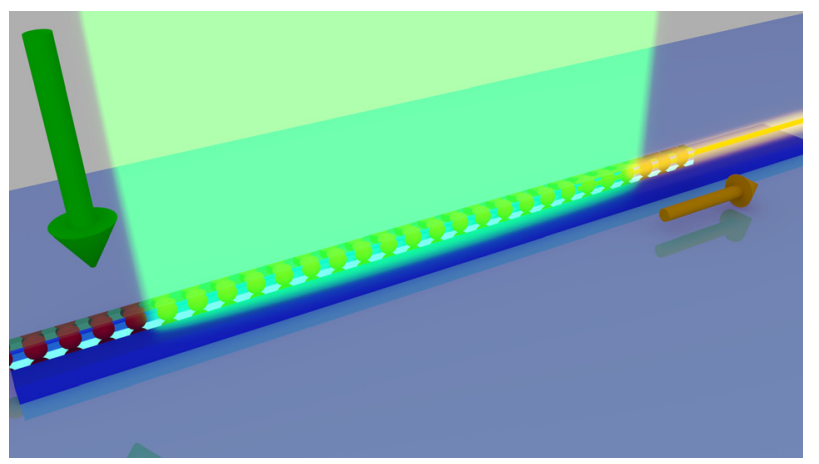

FIG. 1. Schematic illustration of the lasing device, comprising microfluidic channels, pump source, and a region of a periodic droplet array filled with a Gain medium. 


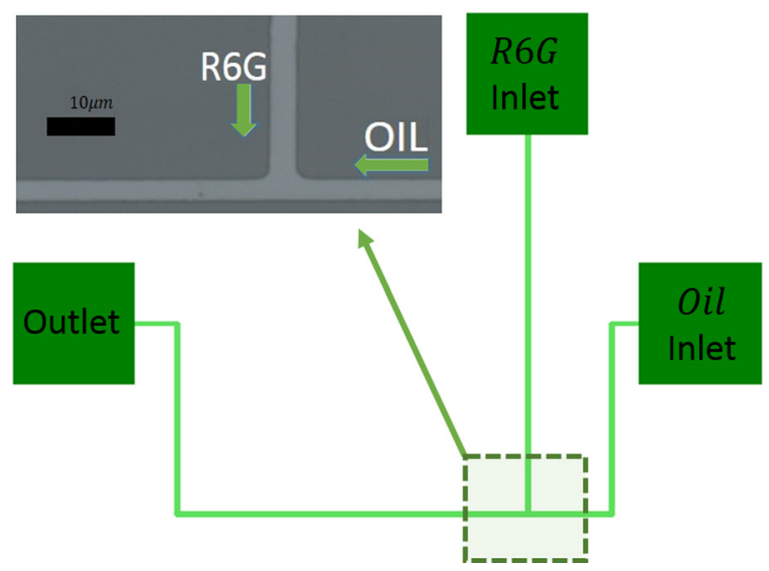

FIG. 2. A schematic top view of the lasing device. The inset shows a micrograph of the fabricated T-junction section in PDMS.

droplets are generated due to immiscibility of oil in the benzyl alcohol. The widths of the benzyl alcohol and oil channels are approximately 1.5 and $1.5 \mu \mathrm{m}$, respectively, whilst their height is $\sim 5 \mu \mathrm{m}$. Each of the two inlets is connected via a Tygon ${ }^{\circledR}$ Tube to a reservoir containing the appropriate liquid. The reservoirs are connected to a pressure regulator (OB1 Elveflow) which independently controls the pressure delivered to each of the channels, with typical pressures of up to 2 bars. The generated unified channel has cross sectional dimensions similar to each of the individual channels $(1.5 \times 5 \mu \mathrm{m})$. In Fig. 3, a representative micrograph of the generated periodic droplet array is shown. The individual droplets and the periodic pattern can be clearly observed. Such a mode of operation has been observed over the course of a few hours, without any evidence of degradation, indicating good stability over time. Furthermore, the standard variation in periodicity is very small (about $0.1 \%$, limited by our imaging capability) which ensures reproducibility and narrow linewidth to be achieved. The period of the droplets as well as the duty cycle can be tuned by controlling the external pressure of the inlets. Typical pressures are in the order of 1 bar.

On top of generating a periodic array in support of the DFB operation, one also needs to confine the light within a waveguide. For this reason, we have used oxygen plasma to bond the PDMS chip which includes the channels and the T-junction to another flat PDMS chip, acting as a cap sealing the liquid core. At the same time, the PDMS serves as the waveguide clad owing to its lower refractive index $(\sim 1.41)$ with respect to the liquid core. Finally, the whole device was bonded to a cover glass via plasma oxygen bonding for the purpose of adding mechanical robustness. Schematic layout showing a cross section of the device can be seen in Fig. 4(a). The calculated fundamental optical mode within the waveguide is shown in Fig. 4(b).

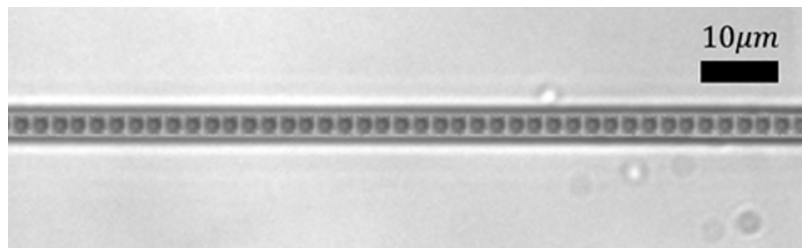

FIG. 3. A representative micrograph of the generated periodic droplet array.

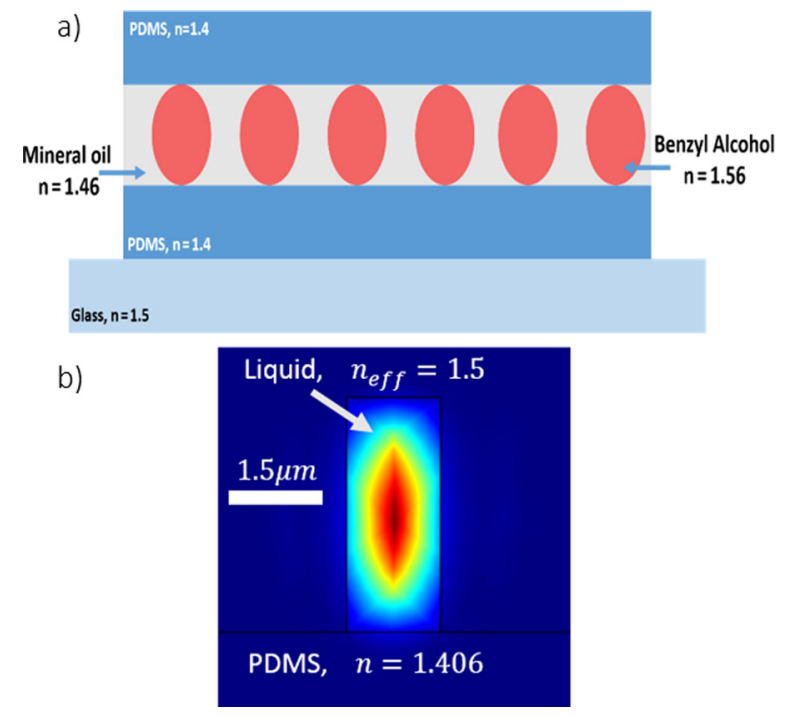

FIG. 4. (a) Schematic side view of the lasing device with refractive index of each component. Typical period is in the range of $1.3-5 \mu \mathrm{m}$. Channel height is about $5 \mu \mathrm{m}$ and its width is about $2 \mu \mathrm{m}$. Generally, the droplets are elliptical in shape. (b) The normalized electric field pattern of in-plane fundamental mode of the liquid waveguide with dimensions of $1.5 \mu \mathrm{m} \times 5 \mu \mathrm{m}$.

Following the device fabrication and operation, we tested its lasing properties. For this purpose, the device was pumped by a frequency doubled Nd:YAG laser (Surelite, Continuum) emitting at $532 \mathrm{~nm}$ and delivering $5 \mathrm{~ns}$ pulses at a $10 \mathrm{~Hz}$ repetition rate. The pump beam was shaped by a cylindrical lens to generate an elongated beam which illuminates the channel from the top. The light collection system consists of an X10 objective, focusing the lasing output emanating from the end of the droplets channel through a notch filter (532 nm Notch Filter, OD6, Edmund Optics) to an optical fiber which is connected to a spectrometer (Ocean Optics 4000 , resolution of $0.22 \mathrm{~nm}$ ).

As mentioned before, the DFB structure serves as our optical feedback mechanism, and thus the lasing wavelength is determined by the Bragg condition ${ }^{28}$

$$
\beta=m \pi / \Lambda,
$$

where $\beta$ is the propagation constant, given by $\beta=2 \pi n_{\text {eff }} / \lambda$, $\lambda$ is the lasing wavelength, $m$ is the Bragg order, $n_{\text {eff }}$ is the effective refractive index of the waveguide, and $\Lambda$ is the period of the droplet array. For the purpose of ensuring single mode operation over the entire bandwidth of the gain medium, one needs to choose the Free Spectral Range (FSR) to be larger than the gain spectral bandwidth. The FSR of the DFB laser is defined as the distance between two neighboring modes: $F S R=\lambda_{m-1}-\lambda_{m}=2 \Lambda n_{g}\left(\frac{1}{m-1}-\frac{1}{m}\right)$, $m=2,3, \ldots$

where $n_{g}$ is the group index. For our device, $n_{g}=n_{\text {eff }}$ $\cong 1.5$, and the grating period can be tuned between $1.3 \mu \mathrm{m}$ and $5 \mu \mathrm{m}$ (at this configuration of the PDMS chip). By using the $1.7 \mu \mathrm{m}$ period, one may achieve an FSR of about $75 \mathrm{~nm}$. Given the fact that R6G supports about $90 \mathrm{~nm}$ (depending on the choice of concentration and dissolved liquid), we can achieve single mode operation, at least around the center of the gain curve. However, when the laser is tuned by increasing the period beyond $1.3 \mu \mathrm{m}$, an additional mode may 
appear. This can be partially overcome by operating at a low gain regime, albeit at the expense of lower output laser power. Reducing the period towards $1.3 \mu \mathrm{m}$ for the purpose of obtaining FSR larger than $100 \mathrm{~nm}$ is possible. However, we observed unstable operation around this regime. A modified design and more elaborated fabrication process may allow to over-come this obstacle. It should also be noted that in the absence of a phase shift, two adjacent modes are expected, yet their spectral separation is orders of magnitude lower than the resolution of our spectrometer and thus such mode splitting was not observed. In Fig. 5, we demonstrate a single mode lasing at $586 \mathrm{~nm}$ with a grating period of $1.74 \mu \mathrm{m}$ (corresponding to 9th Bragg order) with measured linewidth of $0.22 \mathrm{~nm}$ (limited by the spectrometer), alongside the I-I curve showing laser threshold of $\sim 15 \mu \mathrm{J} / \mathrm{mm}^{2}$. As previously mentioned, the tunability of our laser is achieved by controlling the period of our droplet array inside the channel. For a fixed channel width of $1.5 \mu \mathrm{m}$, we could control the period and achieve stable droplet arrays for a long period of time, simply by changing the pressure of the inlets via computer controlled pressure regulators. Indeed, we could obtain a laser output for a wide range of wavelengths ranging from $572.9 \mathrm{~nm}$ to $642.1 \mathrm{~nm}$, i.e., $69.2 \mathrm{~nm}$ of tunability (nearly the entire gain bandwidth of R6G), in a single mode operation. Lasing at several different wavelengths is demonstrated in Fig. 6 . The tunability was achieved by controlling the pressure of the inlets, using a computer controlled pressure regulator. Specifically, we kept the pressure of the R6G channel fixed (1.3 Bar), while continuously changing the pressure of the main channel (oil channel) between $800 \mathrm{mBar}$ (corresponding to a grating period of $1.71 \mu \mathrm{m}$ ) to $1200 \mathrm{mBar}$ (corresponding to a grating period of $1.91 \mu \mathrm{m}$ ). The Droplet diameter increases with the increasing pressure in the oil channel from $\sim 0.85 \mu \mathrm{m}$ to $1 \mu \mathrm{m}$.

In conclusion, we have demonstrated a tunable optofluidic laser in the visible spectral range. The laser is realized by constructing a periodic droplet array within a microfluidic channel. The array provides the DFB mechanism and supports lasing at a specific desired wavelength. By tuning the array period via controlling the pressures in the microfluidic

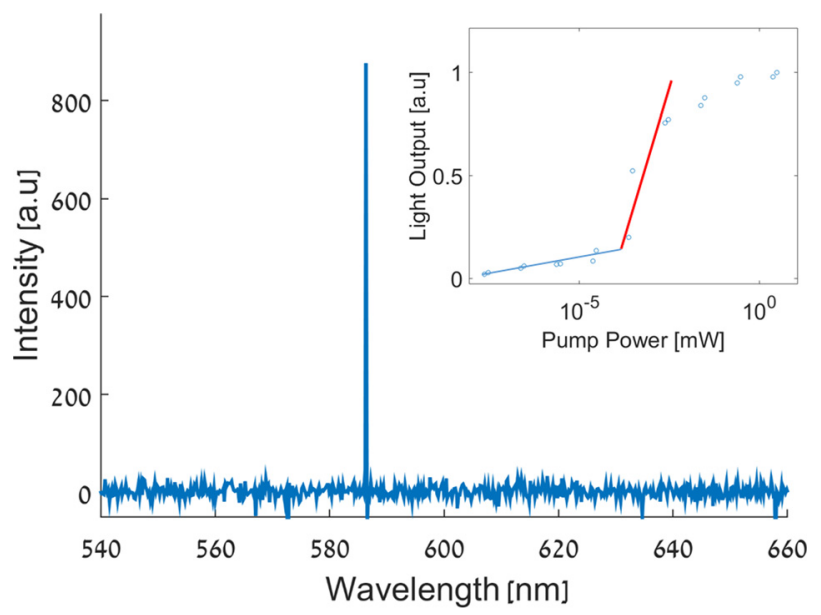

FIG. 5. Typical single mode lasing at $586 \mathrm{~nm}$ with a grating period of $1.74 \mu \mathrm{m}$ (corresponding to 9th Bragg order) with measured linewidth of $0.22 \mathrm{~nm}$, limited by the spectrometer. Inset shows a typical pump light VS light output, laser threshold about $10^{-4} \mathrm{~mW}$.

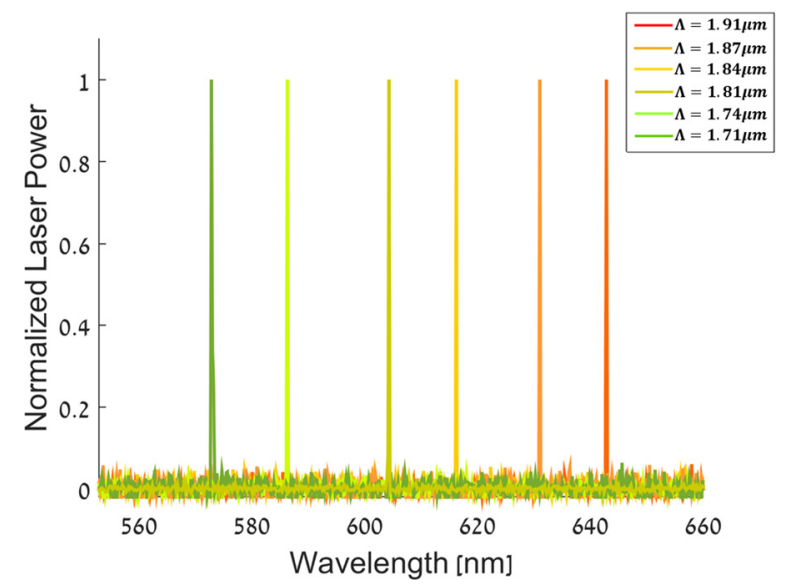

FIG. 6. Tuning of the laser output by changing the grating pitch. Each line represents the laser output from different grating period, as noted by the legend.

channel, we can achieve wavelength tunability of about $70 \mathrm{~nm}$. The demonstrated tunable laser may be used in lab on a chip applications, e.g., as part of an on chip spectrometer.

${ }^{1}$ C. Monat, P. Domachuk, and B. J. Eggleton, Nat. Photonics 1, 106 (2007).

${ }^{2}$ D. Psaltis, S. R. Quake, and C. Yang, Nature 442, 381 (2006).

${ }^{3}$ U. Levy and R. Shamai, Microfluid. Nanofluid. 4, 97 (2007).

${ }^{4}$ Y. Fainman, L. P. Lee, D. Psaltis, and C. Yang, Optofluidics: Fundamentals, Devices, and Applications, 1st ed. (McGraw-Hill Education, New York, 2009).

${ }^{5}$ L. Pang, H. M. Chen, L. M. Freeman, and Y. Fainman, Lab Chip 12, 3543 (2012).

${ }^{6}$ T. Wienhold, F. Breithaupt, C. Vannahme, M. B. Christiansen, W. Dörfler, A. Kristensen, and T. Mappes, Lab Chip 12, 3734 (2012).

${ }^{7}$ B. Helbo, A. Kristensen, and A. Menon, J. Micromech. Microeng. 13, 307 (2003).

${ }^{8}$ M. Gersborg-Hansen and A. Kristensen, Appl. Phys. Lett. 89, 103518 (2006).

${ }^{9}$ C. Peroz, J.-C. Galas, L. Le Gratiet, Y. Chen, and J. Shi, Appl. Phys. Lett. 89, 243109 (2006).

${ }^{10}$ W. Song, A. E. Vasdekis, Z. Li, and D. Psaltis, Appl. Phys. Lett. 94, 51117 (2009).

${ }^{11}$ M. Gersborg-Hansen and A. Kristensen, Opt. Express 15, 137 (2007).

${ }^{12}$ C. Vannahme, C. L. C. Smith, M. Brøkner Christiansen, and A. Kristensen, Appl. Phys. Lett. 101, 151123 (2012).

${ }^{13}$ W. Song, A. E. Vasdekis, Z. Li, and D. Psaltis, Appl. Phys. Lett. 94, 161110 (2009).

${ }^{14}$ H. Taniguchi and S. Tanosaki, Jpn. J. Appl. Phys., Part 2 32, L1421 (1993).

${ }^{15}$ M. Saito and K. Koyama, J. Opt. 14, 065002 (2012).

${ }^{16}$ M. Humar and S. Hyun Yun, Nat. Photonics 9, 572 (2015).

${ }^{17}$ H. Azzouz, L. Alkhafadiji, S. Balslev, J. Johansson, N. A. Mortensen, S. Nilsson, and A. Kristensen, Opt. Express 14, 4374 (2006).

${ }^{18}$ J. D. Suter, Y. Sun, D. J. Howard, J. A. Viator, and X. Fan, Opt. Express 16, 10248 (2008).

${ }^{19}$ Y. Chen, Z. Li, Z. Zhang, D. Psaltis, and A. Scherer, Appl. Phys. Lett. 91, 051109 (2007).

${ }^{20}$ T. Grossmann, T. Wienhold, U. Bog, T. Beck, C. Friedmann, H. Kalt, and T. Mappes, Light Sci. Appl. 2, e82 (2013).

${ }^{21}$ G. Aubry, Q. Kou, J. Soto-Velasco, C. Wang, S. Meance, J. J. He, and A. M. Haghiri-Gosnet, Appl. Phys. Lett. 98, 111111 (2011).

${ }^{22}$ S. K. Y. Tang, Z. Li, A. R. Abate, J. J. Agresti, D. A. Weitz, D. Psaltis, and G. M. Whitesides, Lab Chip 9, 2767 (2009).

${ }^{23}$ Z. Li, Z. Zhang, A. Scherer, and D. Psaltis, Opt. Express 14, 10494 (2006).

${ }^{24}$ S. K. Y. Tang, C. A. Stan, and G. M. Whitesides, Lab Chip 8, 395 (2008).

${ }^{25}$ Y. Aoyagi, T. Aoyagi, K. Toyoda, and S. Namba, Appl. Phys. Lett. 27, 687 (1975).

${ }^{26}$ T. Beatus, R. H. Bar-Ziv, and T. Tlusty, Phys. Rep. 516, 103 (2012).

${ }^{27}$ L. Stern, A. Bakal, M. Tzur, M. Veinguer, N. Mazurski, N. Cohen, and U. Levy, Sensors (Basel). 14, 16799 (2014).

${ }^{28}$ H. Kogelnik, Appl. Phys. Lett. 18, 152 (1971). 